\title{
The ionospheric response during an interval of Pc5 ULF wave activity
}

\author{
M. Lester, J. A. Davies, T. K. Yeoman \\ Department of Physics and Astronomy, University of Leicester, Leicester, LE1 7RH, UK \\ Received: 21 June 1999 / Revised: 29 September 1999 / Accepted: 4 October 1999
}

\begin{abstract}
A preliminary analysis of Pc5, ULF wave activity observed with the IMAGE magnetometer array and the EISCAT UHF radar in the post midnight sector indicates that such waves can be caused by the modulation of the ionospheric conductivity as well as the wave electric field. An observed Pc5 pulsation is divided into three separate intervals based upon the EISCAT data. In the first and third, the Pc5 waves are observed only in the measured electron density between 90 and $112 \mathrm{~km}$ and maxima in the electron density at these altitudes are attributed to pulsed precipitation of electrons with energies up to $40 \mathrm{keV}$ which result in the height integrated Hall conductivity being pulsed between 10 and $50 \mathrm{~S}$. In the second interval, the Pc5 wave is observed in the F-region ion temperature, electron density and electron temperature but not in the $\mathrm{D}$ and $\mathrm{E}$ region electron densities. The analysis suggests that the wave during this interval is a coupled Alfven and compressional mode.
\end{abstract}

Key words: Ionosphere (electric fields and currents) Magnetospheric physics (magnetosphere-ionosphere interaction; MHD waves and instabilities).

\section{Introduction}

Observations of ULF waves by coherent radars such as STARE and SABRE are many (e.g. Walker et al., 1979; Allan et al., 1982; Yeoman et al., 1990). From such observations, the electric field of the wave in the ionosphere could be measured for the first time (Walker et al., 1979). Observations of waves with large azimuthal wave numbers, previously difficult to observe with ground based magnetometers, could also be made with these radars (Allan et al., 1982). Furthermore, a method

Correspondence to: M. Lester for identifying the "compressiveness" of ULF waves was discussed by Yeoman et al. (1990). There are, however, few simultaneous observations of the variations in the ionospheric electron density, electron temperature and ion temperature during ULF wave activity (Doupnik et al., 1977; Crowley et al., 1985, 1987; Lathuillere et al., 1986; Buchert et al., 1999). Such observations are important to determine the ULF wave mode and the nature of the damping mechanism for ULF waves amongst other things. In this letter we present a preliminary analysis of observations by EISCAT during ULF waves in the interval 0200-0500 UT on April 21, 1993.

\section{Magnetometer data}

Band-pass filtered data, between 20 and $500 \mathrm{~s}$, from four stations of the IMAGE array, Tromsø, Kilpisjarvi, Masi and Kevo, for the interval of interest are given in Fig. 1. Between 0230 and 0450 UT there appears to be a single Pc5 ULF wave, which is composed of several different wave packets, all of a similar frequency. The interval can be divided into three distinct parts (see also later), between 0230 and 0315 UT, 0315-0410 UT and 04100500 UT. The $Y$, geographic east-west, component (Fig. 1b) is the strongest component in the first interval, while the $X$, geographic north-south, (Fig. 1a) and $Y$ components are comparable during the second interval and the $X$ component is the largest in the third interval. Spectral analysis of the data from all IMAGE stations indicates that the dominant frequency in both $X$ and $Y$ components is $4.8 \mathrm{mHz}, 5.2 \mathrm{mHz}$ and $5.3 \mathrm{mHz}$ in the first, second and third intervals, respectively. Note that the resolution of these frequencies is $\pm 0.4 \mathrm{mHz}$. Comparison of phase across the four stations in Fig. 1 provides an estimate of the azimuthal wave numbers, or $m$ values. The outermost stations, TRO and KEV, have the smallest separation in latitude, $0.3^{\circ}$, thereby reducing any effects caused by latitudinal phase gradients, and are separated by $6.3^{\circ}$ in longitude. Based upon these stations, the $X$ and $Y$ component $m$ values for the 


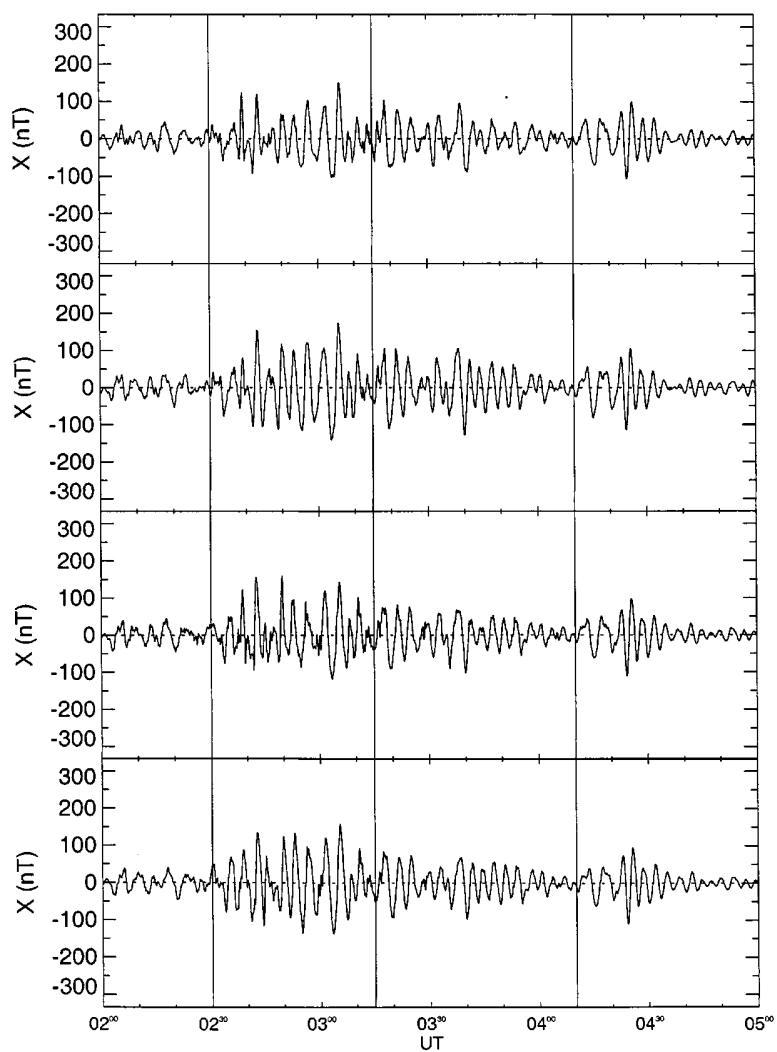

Fig. 1a, b. Band-pass filtered magnetometer data between 0200 and 0500 UT from Tromso (TRO), Kilpisjarvi (KIL), Masi (MAS) and Kevo (KEV). The filter bandwidth is 20 to $500 \mathrm{~s}$. The $X$ components

dominant spectral component in each interval range between 13 and 18 (i.e. westward).

\section{EISCAT data}

During the interval, the EISCAT radar was operating in common mode CP-1-K which is a simple mode whereby the Tromsø UHF radar points in a direction which is approximately aligned along the magnetic field direction in the F-region. The two remote receiver beams intersect the Tromsø beam at an altitude of $278 \mathrm{~km}$. The mode and experimental codes are described in detail elsewhere (e.g. Davies et al., 1997). In this brief work we present both long pulse estimates of electron density and temperature and ion temperature at altitudes above $150 \mathrm{~km}$, with a resolution of $22 \mathrm{~km}$ along the beam, and height integrated Hall and Pedersen conductivities derived from raw electron density measurements, or power profile, between 70 and $400 \mathrm{~km}$ at a resolution of $4.5 \mathrm{~km}$. The Hall and Pedersen conductances have been calculated following the method outlined in Lester et al. (1996). The tristatic measurements of ion velocity at $278 \mathrm{~km}$ allow the calculation of the electric field.

Representative time series of electron density, $N_{e}$, electron temperature, $T_{e}$, ion temperature, $T_{i}$, at an altitude of $\sim 212 \mathrm{~km}$, the east-west, $V_{E}$, and north-south, $V_{N}$, components of the ion velocity at $278 \mathrm{~km}$, and the

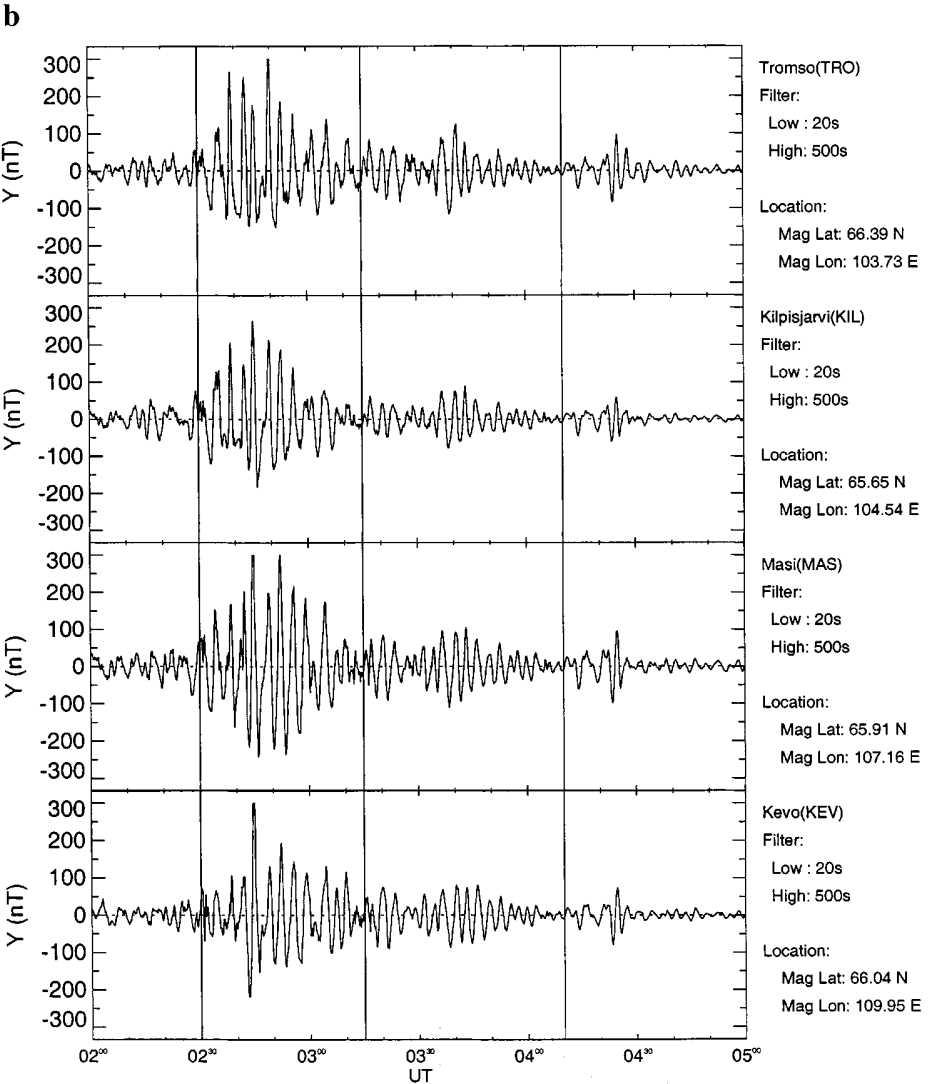

(north-south) are in a and the $Y$ components (east-west) are in $\mathbf{b}$. The vertical lines identify the three separate intervals of ULF wave activity as described in the text

height integrated Hall conductivity, $\Sigma_{H}$, for the interval 0200 to 0500 UT are presented in Fig. 2a. The electron density, electron temperature, ion temperature and $\Sigma_{H}$ are plotted with a $10 \mathrm{~s}$ integration, while the ion velocity data are plotted with a $20 \mathrm{~s}$ integration. Vertical lines are drawn to indicate the three separate intervals identified. The F-region measurements only respond to the wave activity between 0315 and 0410 UT, while there is periodic behaviour in $\Sigma_{H}$ during both the first and third intervals but not the second.

In interval $1,0230-0315 \mathrm{UT}, N_{e}$ at $212 \mathrm{~km}$ is typically $1 \times 10^{11} \mathrm{~m}^{-3}$. At this altitude $T_{e}$ varies between 1200 and $1400 \mathrm{~K}$ and $T_{i}$ between 800 and $1200 \mathrm{~K}$. The larger ion temperatures are related to the enhanced ion velocities and are caused by ion frictional heating (e.g. Davies et al., 1997). The lower E-region electron density (data not shown) is $10^{11} \mathrm{~m}^{-3}$ between 0210 and 0230 UT, but during the following $45 \mathrm{~min}$ it varies periodically between $5 \times 10^{10} \mathrm{~m}^{-3}$ and $5 \times 10^{11} \mathrm{~m}^{-3}$. A dominant spectral peak near $5.2 \mathrm{mHz}$ is evident in the spectral analysis of the E-region electron density leads to the periodic variation in $\Sigma_{H}$ between 10 and $50 \mathrm{~S}$, shown in Fig. 2a, while the height integrated Pedersen conductivity, $\Sigma_{p}$, varies between 5 and $10 \mathrm{~S}$.

During interval 2 there are clear periodic variations in F-region electron density, electron temperature and ion temperature. The maxima in $N_{e}$, about $3 \times 10^{11} \mathrm{~m}^{-3}$, are the highest values at these altitudes 
a

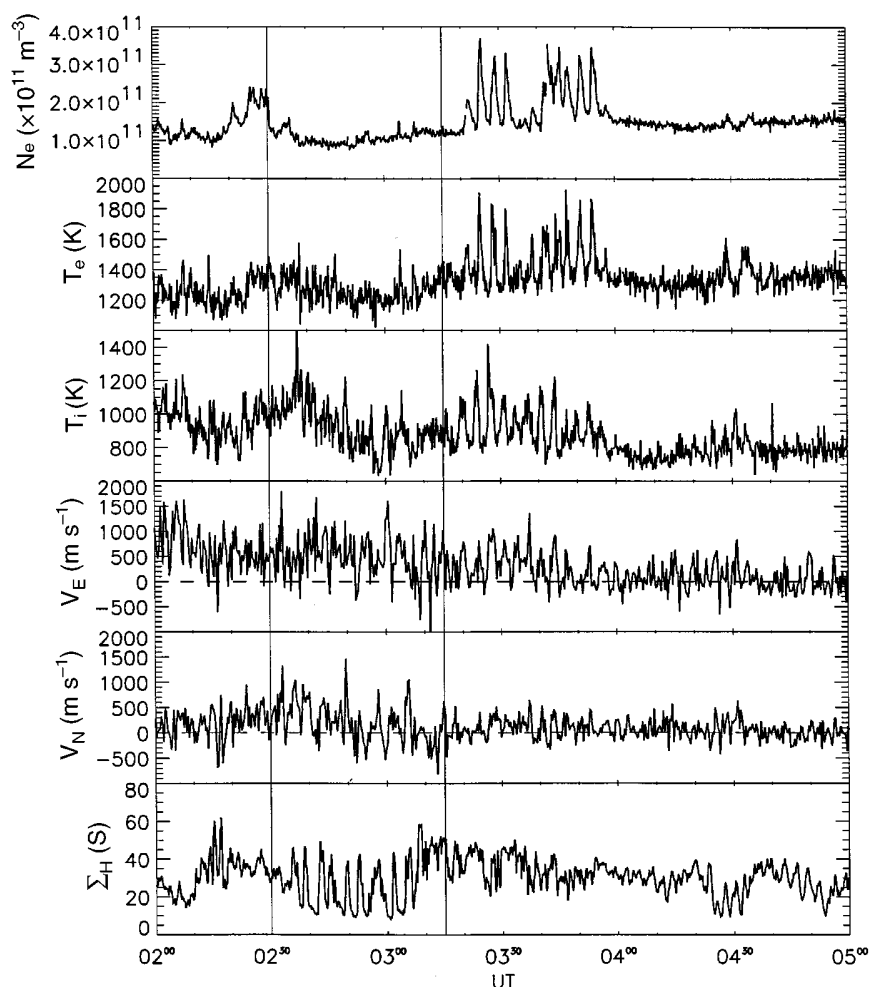

Fig. 2a, b. The EISCAT parameters, electron density at $212.5 \mathrm{~km}$, electron temperature at $212.5 \mathrm{~km}$, ion temperature at $212.5 \mathrm{~km}$, the east-west component of the ion velocity at $278 \mathrm{~km}$, the north-south component of the ion velocity at $278 \mathrm{~km}$ and the height integrated

during the whole interval. Similarly, the peak values in $T_{e}$ of $1800 \mathrm{~K}$ also represent the largest values at this altitude during the interval. Variations in $T_{i}$ are at least $200 \mathrm{~K}$ larger than the background values, and typically peak at $1200 \mathrm{~K}$, while the variations in the ion velocity appear largest in the east-west component, with peak values of $+1.0 \mathrm{~km} \mathrm{~s}^{-1}$, which means the ion velocity is enhanced in the eastward direction. There are no periodic variations in $\Sigma_{H}$, although the values are larger than at almost any other time during the whole interval. Spectral analysis of $N_{e}, T_{e}, T_{i}, V_{E}, V_{N}$ and the magnitude of the ion velocity indicates a dominant spectral peak of $5.1 \mathrm{mHz}$ in all parameters.

During the final wave packet observed by the magnetometer data, the F-region parameters are fairly constant throughout the interval with no sign of the periodicities seen earlier. There are, however, clear periodic variations in $\Sigma_{H}$ between 0420 and 0500 UT with no similar variations in $\Sigma_{P}$. The dominant spectral peak in the E-region electron density is $5.3 \mathrm{mHz}$.

The EISCAT measurements during the second of the wave packets demonstrate the relative variations in the F-region ionospheric parameters during the ULF wave activity. Figure $2 \mathrm{~b}$ is in the same format as Fig. $2 \mathrm{a}$ but covers the interval 0315 UT to 0410 UT. There are two separate packets of pulsed behaviour in electron density and temperature. Initially there are four peaks in $N_{e}$ and $T_{e}$ between $0320 \mathrm{UT}$ and $0334 \mathrm{UT}$, and a further six pulses in the second packet. On the other hand, b

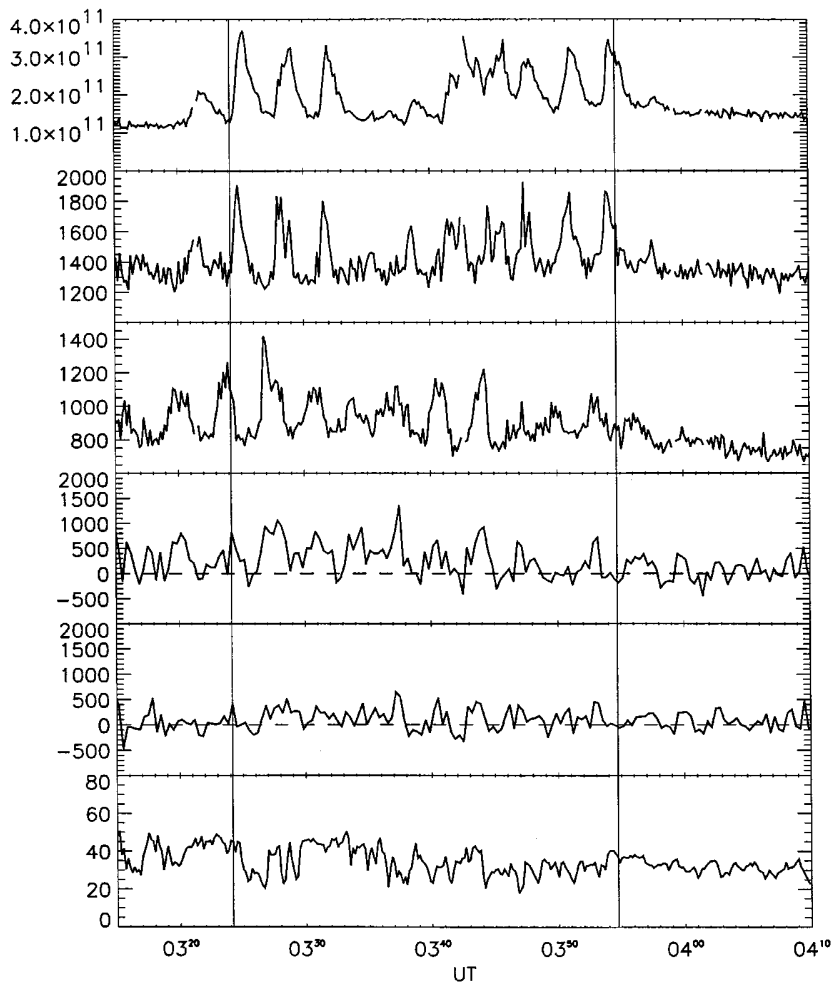

conductivity Hall conductivity. a covers the time interval 0200 to 0500 UT and $\mathbf{b}$ the interval 0315 to 0410 UT. The vertical lines in a identify the three separate intervals of ULF wave activity as described in the text

$T_{i}$ appears to be pulsed throughout the period up to 0400 UT.

The first signature of the wave activity occurs at 0319 UT when $T_{i}$ increases from $800 \mathrm{~K}$ to more than $1000 \mathrm{~K}$. The pulse of enhanced $T_{i}$ lasts for approximately $120 \mathrm{~s}$ and is followed by an interval of unperturbed ion temperature. This behaviour continues and the ion temperature has the appearance of a square wave. During this pulse of enhanced ion temperature, $N_{e}$ and $T_{e}$ are low and become enhanced as $T_{i}$ decreases. Normally $T_{e}$ peaks just before or at the same time as $N_{e}$ and also begins to decrease before $N_{e}$. In contrast to $T_{i}$, the pulses in $N_{e}$ and $T_{e}$ have rapid rise times to a peak value with subsequent slower decay times. A cross correlation analysis of the three times series indicates that the ion temperature and electron density are exactly $180^{\circ}$ out of phase while the electron temperature leads the electron density by about $40^{\circ}$. This latter phase difference is a result of the decrease in electron temperature leading that in electron density.

\section{Discussion}

The Pc5 wave activity observed in the magnetometer data can be divided into three separate intervals as a result of the analysis of the EISCAT data. In the first and third intervals, the only response in the EISCAT observations is in the lower E-region and D-region 
electron density, while in the second interval the response is seen in the F-region but not in the lower E- or D-regions. For the purpose of this work we discuss separately the wave activity in the first and second intervals.

A careful study of the electron density as a function of altitude during the first interval (data not shown) indicates that the wave occurs in the electron density measured between $90 \mathrm{~km}$ and $112 \mathrm{~km}$ altitude, with the largest variations in density at $\sim 99 \mathrm{~km}$. At this altitude the peak density is $5 \times 10^{11} \mathrm{~m}^{-3}$, with a minimum density of $5 \times 10^{10} \mathrm{~m}^{-3}$. The cause of such pulsed behaviour in electron density is most likely pulsed particle precipitation since such densities are higher than expected for this local time, $\sim 0430$ LT, in April. It is instructive to consider the energy of the particles which would cause such ionisation at these altitudes. For a monoenergetic, field-aligned beam of electrons of $20 \mathrm{keV}$ the peak ionisation rate occurs near $100 \mathrm{~km}$ (Rees, 1989), while a beam of electrons of $40 \mathrm{keV}$ has a peak ionisation rate near $90 \mathrm{~km}$. A detailed inversion of the ionisation profile would provide a more accurate energy spectrum but is not attempted here. This variation in D-region and lower E-region densities leads to the conductance varying between 10 and $50 \mathrm{~S}$.

Recently, Buchert et al. (1999) have reported observations of a ULF wave in the afternoon sector during which the conductance varied by a factor of 2 . These authors concluded that the cause of the conductance variations was pulsed electron precipitation in the energy range $2-5 \mathrm{keV}$. The electrons which caused the conductance variations presented in Fig. 1 are of much higher energy than those reported by Buchert et al. (1999). The $\mathrm{m}$ value of the event discussed by Buchert and colleagues is 30 , larger than that of the wave in Fig. 1, while the direction of phase propagation is westward for both events. However as the local time of the event discussed here is pre-dawn, a westward phase propagation is consistent with the wave being driven by the solar wind as it passes over the magnetopause and by ions drifting in the magnetosphere. Thus, it is not possible to distinguish between these two driving mechanism, as in the case of Buchert et al. (1999). It is also worth noting that no significant solar or substorm activity took place at the time of either observation, although a substorm did occur a few hours before the case reported here.

Moving to interval two, the first point to discuss is the change in the E-region density. The density at $99.4 \mathrm{~km}$ varies between 2 and $5 \times 10^{11} \mathrm{~m}^{-3}$, starting at the higher value and gradually decreasing, although not monotonically. Again this density is higher than expected at these altitudes at this local time and is also higher than the density at higher altitudes. For example, at $112 \mathrm{~km}$ the density has a maximum of $3 \times 10^{11} \mathrm{~m}^{-3}$ and is always lower than the values at $99 \mathrm{~km}$. Secondly, there is no clear signature in the Eregion density of the ULF wave activity seen in the magnetometer data or in the F-region ionospheric parameters. As discussed already the likely cause of the enhanced $\mathrm{D}$ region and lower $\mathrm{E}$ region ionisation is particle precipitation and on this occasion it appears to be more continuous, if variable, rather than pulsed. The high electron densities lead to values in $\Sigma_{H}$ which vary between 20 and $50 \mathrm{~S}$.

The phase relationships between $N_{e}, T_{e}$ and $T_{i}$ represent the other intriguing aspect of this interval. The simultaneous enhancements in electron density and temperature are likely to be caused by particle precipitation, while the ion temperature enhancements are caused by enhancements in the ion velocity, most easily seen in the eastward component (Fig. 2). The latter enhancements are possibly caused by the ULF wave electric field. Of the ULF wave modes, the Alfven mode has a significant electric field in the ionosphere (e.g. Yeoman et al., 1990). Observations by SABRE demonstrated that ULF waves are found to be increasingly compressional as the aziumthal wave number increased, at least up to a value of 12 . The magnetometer observations discussed earlier indicate that the wave numbers are $>13$, suggesting that the wave observed during the second interval had a significant compressional component as well as an Alfvenic component. Furthermore, the wave frequency of $5.2 \mathrm{mHz}$ during this interval is close to the expected resonant frequency for the latitude of Tromsø (e.g. Mathie et al., 1999).

The high $\Sigma_{H}$ indicates continuing precipitation of high energy particles which is not pulsed as in the first and third intervals. The particles causing the enhanced $\mathrm{D}$ and lower E-region electron densities are of much higher energy than those which are believed to be causing the enhancements in the F-region electron density and temperature. Why the higher energy part of the spectrum is not pulsed is unclear, however.

\section{Conclusions}

We report joint observations of Pc5 pulsations by the IMAGE magnetometer array and EISCAT. Such observations are rare, although with improvements to the receiver sensitivity at Tromsø we anticipate that more events will be observed. The interval of ULF wave activity examined here has two different responses in the ionosphere. In the first part of the interval, only enhancements in lower $\mathrm{E}$ region and $\mathrm{D}$ region electron densities are observed. We suggest these are caused by pulsed particle precipitation at energies of order 20 $40 \mathrm{keV}$. Furthermore, the ground magnetic signature may simply be a response to the changing Hall conductivity. In the second interval a complicated phase relationship between the ion temperature and electron density and temperature exists. There are two processes occurring, one associated with the wave electric field and the other with the pulsed precipitation of low energy particles. The joint observations of the ULF wave electric field and high wave number indicates that the wave consists of both Alfven and fast modes. Simultaneously, there continues to be precipitation of high energy particles, $20-40 \mathrm{keV}$, in order to maintain the high Hall conductance. The preliminary analysis under- 
taken here cannot distinguish whether this is solely a temporal variation or solely a spatial variation. The interval does clearly respond in two different ways during the period of ULF wave activity, without any clear difference in the ground magnetic signatures at the time. Further detailed modelling and analysis of this event is required and will be presented in a future study.

Acknowledgements. EISCAT is funded by the research agencies of Finland, France, Germany, Japan, Norway, Sweden and the United Kingdom. The IMAGE magnetometer data used in this work were collected as a German-Finnish-Norwegian-Polish project conducted by the Technical University of Braunschweig, Germany.

The Editor-in-chief thanks F. Menk for his help in evaluating this paper.

\section{References}

Allan, W., E. M. Poulter, and E. Nielsen, STARE observations of a Pc5 pulsation with large azimuthal wave numbers, J. Geophys. Res., 87, 6163-6172, 1982.

Buchert, S. C., R. Fujii, and K.-H. Glassmeier, Ionospheric conductivity modulation in ULF pulsations, J. Geophys. Res., 104, 10 119-10 133, 1999.

Crowley, G., N. Wade, J. A. Waldock, T. R. Robinson, and T. B. Jones, High time-resolution observations of periodic frictional heating associated with a Pc5 micropulsation, Nature, 316, 528, 1985.

Crowley, G., W. J. Hughes, and T. B. Jones, Observational evidence of cavity modes in the Earth's magnetosphere, J. Geophys. Res., 92, 12 233-12 240, 1987.

Davies, J. A., M. Lester, and I. W. McCrea, A statistical study of ion frictional heating observed by EISCAT, Ann. Geophysicae., 15, 1399-1411, 1997.

Doupnik, J. R., P. M. Banks, and A. Brekke, Incoherent scatter radar observations during three sudden commencements and a Pc5 event on August 4, 1972, J. Geophys. Res., 82, 499-514, 1977.

Lathuillere, C., F. Glangeaud, and Z. Y. Zhao, Ionospheric ion heating by ULF Pc5 magnetic pulsations, J. Geophys. Res., 91, 1619-1626, 1986.

Lester, M., J. A. Davies, and T. S. Virdi, High latitude Hall and Pedersen conductances during substorm activity in the SUNDIAL-ATLAS Campaign, J. Geophys. Res., 101, 26719 $26728,1996$.

Mathie, R. A., F. W. Menk, I. R. Mann, and D. Orr, Discrete field line resonances and the Alfvén continuum in the outer magnetosphere, Geophys. Res. Lett., 26, 659-662, 1999.

Rees, M. H., Physics and chemistry of the upper atmosphere, Cambridge University Press, Cambridge, UK, 1989.

Walker, A. D. M., R. A. Greenwald, W. F. Stuart, and C. A. Green, STARE auroral radar observations of Pc5 geomagnetic pulsations, J. Geophys. Res., 84, 3373-3308, 1979.

Yeoman, T. K., M. Lester, D. Orr, and H. Lühr, Ionospheric boundary conditions of hydromagnetic waves: the dependence on azimuthal wave number and a case study, Planet. Space Sci., 38, 1315-1325, 1990. 